\title{
Saúde e Humanização do Contexto Organizacional sob a Perspectiva da Análise do Comportamento
}

\author{
Healt and Humanization Under the Perspective of Behavior Analysis
} Salud y Humanización del Contexto Organizacional Bajo la Perspectiva del Análisis del
Comportamiento

\author{
Eveli Freire Vasconcelos ${ }^{1}$ \\ Universidade Católica Dom Bosco (UCDB) \\ Gerson Armênio Fabrício Bauermeister \\ Assembleia Legislativa do Estado de Mato Grosso \\ Heloisa Bruna Grubits Freire \\ Universidade Católica Dom Bosco (UCDB) \\ Thaize de Souza Reis \\ Universidade Federal de Mato Grosso do Sul (UFMS)
}

\begin{abstract}
Resumo
O artigo discute a aplicação dos princípios da Análise do Comportamento no contexto organizacional e do trabalho, que tem sido identificada pela sigla Organizational Behavior Management (OBM). Destaca-se a concepção comportamental de autocontrole, bem como as técnicas comportamentais para alcançá-lo, pois se entende que o autocontrole tem estreita relação com a promoção de saúde em diversos ambientes, incluindo o organizacional. São também apresentados conceitos como inflexibilidade e flexibilidade psicológica, que ajudam a entender a gênese e manutenção de comportamentos ditos psicopatológicos e saudáveis, respectivamente. Os conteúdos apresentados conduzem a uma discussão quanto à posição ocupada pelo OBM nos contextos organizacionais e em como os profissionais contribuem para que o performer seja visto como investimento, em lugar de matéria-prima. Nessa direção, a promoção do autocontrole é apresentada como uma das razões pelas quais o OBM deve ser encarado como uma aproximação mais humana às organizações.
\end{abstract}

Palavras-chave: organizações, trabalho, saúde, autocontrole, análise do comportamento

\begin{abstract}
This paper addresses Behavior Analysis applied in the organizational and work context, which has been identified as Organizational Behavior Management (OBM). It emphasizes the behavioral conception of self-control, as well as the behavioral techniques to reach it, because the close relation between self-control and health promotion in diverse environments, including organizations. Concepts such as psychological inflexibility and flexibility are also presented. These concepts help to understand the genesis and maintenance of so-called psychopathological and healthy behaviors. The contents presented lead to a discussion about the position occupied by OBM in organizational contexts and how psychologists could contribute to view the performer as an investment rather than a raw material. In this direction, the promotion of self-control is presented as one of the reasons why OBM should be considered a more humane approach to organizations.

Keywords: organizations, work, health, self-control, organizational behavior management

\section{Resumen}

El artículo discute la aplicación del análisis del comportamiento, en un contexto organizacional de trabajo, el cual ha sido identificado por la sigla OBM (de Organizational Behavior Management). Se destaca la concepción comportamental de autocontrol, bien como las técnicas comportamentales para alcanzarlo, pues se entiende que el autocontrol tiene estrecha relación con la salud en diversos ambientes, incluyendo el organizacional. Son presentados también conceptos, tales como inflexibilidad y flexibilidad psicológicas, que ayudan a entender la génesis y la mantención de los comportamientos dichos psicopatológico y saludable, respectivamente. Los contenidos presentados conducen a una discusión en cuanto a la
\end{abstract}

\footnotetext{
${ }^{1}$ Endereço de contato: Universidade Católica Dom Bosco, Clínica de Psicologia, Av. Tamandaré, 6.000, Jd. Seminário. CEP 79117-900, Campo Grande, MS, Brasil. E-mail: evelivasconcelos@yahoo.com.br
} 
posición ocupada por la OBM en el contexto organizacional y cómo los profesionales contribuyen para que el performer sea vista como inversión en vez de materia prima. En ese sentido, la promoción del autocontrol es presentada como una de las razones por las cuales la OBM debería de ser encarada como una aproximación más humana frente a las organizaciones.

Palabras clave: organizaciones, trabajo, salud, autocontrol, análisis del comportamiento

\section{Introdução}

Este artigo aborda o papel da aplicação dos princípios da Análise Experimental do Comportamento no contexto organizacional e do trabalho e suas consequências à saúde do trabalhador. Destaca-se a concepção comportamental de autocontrole, bem como as técnicas comportamentais para alcançá-lo, pois se entende que o autocontrole tem estreita relação com a promoção de saúde em diversos ambientes, incluindo o organizacional.

A sigla OBM (Organizational Behavior Management) refere-se à aplicação dos princípios da Análise Experimental do Comportamento no contexto das organizações e do trabalho. Há uma argumentação quanto à posição ocupada pelo OBM na humanização dos contextos organizacionais e em como os profissionais de OBM contribuem para que o trabalhador seja visto como investimento. As consequências dessa argumentação alcançam não apenas a relativa obscuridade em que se encontra o OBM quando comparado à Psicologia OrganizacionalIndustrial tradicional, mas também a marginalização da Análise do Comportamento em geral quando comparada ao mainstream da Psicologia, sobretudo em relação à Psicologia Cognitiva. Assim, a concepção comportamental de autocontrole e as técnicas para sua promoção são apresentadas como uma das razões pelas quais o OBM pode ser encarado como uma aproximação mais humana às organizações.

O que está em questão é refletir criticamente sobre propostas que contribuam com o desenvolvimento de estratégias voltadas à saúde dos trabalhadores, considerando que o contexto de trabalho conforme discorrido por Zanelli (2016) está na base de fatores multideterminantes de proteção ou perda da saúde.

\section{Autocontrole e Promoção da Saúde}

Fala-se na necessidade de autocontrole quando um determinado comportamento tem alta probabilidade de ocorrência e apresenta consequências conflitantes, como contingências reforçadoras a curto prazo e aversivas a longo prazo ou aversivas a curto prazo e reforçadoras a longo prazo. Um exemplo do primeiro tipo seria a ingestão constante de alimentos gordurosos, o que pode ser reforçador a curto prazo, mas a longo prazo pode acarretar problemas de saúde. Fazer dieta parece ilustrar uma contingência que pode ter caráter aversivo a curto prazo, porém reforçador a longo prazo. Para Skinner (1953), autocontrole envolve manipular variáveis ambientais de forma a alterar a probabilidade de emissão da resposta a ser controlada. No primeiro exemplo mencionado, o autocontrole seria a emissão de respostas que diminuíssem a probabilidade de ingestão de alimentos gordurosos, tais como evitar situações em que tais alimentos estarão disponíveis.

As técnicas de autocontrole, por si mesmas, e combinadas com outros tratamentos são eficazes para tratar problemas de saúde, como desordens alimentares, ludopatia, vício em cigarro, diabetes, tricotilomania, consumo de drogas, obesidade e asma, dentre outras (Rosa, 
Olivares \& Sánches, 1999 ). Dito isso e considerada a emergência da saúde do trabalhador como um tema cada vez mais estudado e necessário para as organizações, é crítico buscar compreender qual a relação entre padrões de autocontrole e uma vida saudável (Jaques, 2003), sobretudo em uma noção de saúde que não envolva apenas os aspectos responsáveis pela patologia, mas que considere os aspectos referentes à saúde.

O modelo construcional, um dos pioneiros na Psicologia Experimental do comportamento, foi utilizado em pesquisas na área de saúde para o tratamento de comportamentos em excessos, como tabagismo e gagueira (Goldiamond, 1974 apud Casseb, Bispo, \& Ferreira, 2008; Kerbauy, 2009). Ao invés de eliminar ou diminuir comportamentos disfuncionais, o modelo construcional trabalha com a construção de repertórios mais adaptáveis. Assim, o repertório adaptativo do cliente, já existente no começo do tratamento, é ampliado por meio do uso da manipulação de contingências comportamentais.

Programas de treinamento em autocontrole têm "como benefício permitir ao indivíduo manter o controle do próprio comportamento e seguir as orientações do tratamento" (Casseb, Bispo, \& Ferreira, 2008, p. 224), pois ele fica em contato com os ganhos obtidos ao longo da intervenção. Doenças crônicas relacionadas com padrões de comportamentos são alvos desse tipo de tratamento. Usar uma técnica de automonitoramento para instituir o autocontrole, por exemplo, permite que a pessoa que se automonitore tenha uma avaliação geral e real de suas atividades, a partir das quais possa se chegar ao autocontrole.

Algumas regularidades têm sido observadas nos treinamentos de autocontrole em questões de saúde, relacionadas a como intervenções menos intensas trazem benefícios superiores a intervenções mais intensas. Também existem evidências que, ao combinar as técnicas de autocontrole com outras estratégias, a eficácia é diminuída, quando comparada a situações em que são aplicadas sozinhas. Ainda assim, esses dados são questionáveis, não sendo possível afirmar se as técnicas de autocontrole devem ou não ser usadas sem combinações com outras. Isso deve ser decidido em função do tipo de questão a ser trabalhada com o indivíduo e não como uma prescrição geral. Portanto, vale a recomendação de que não se deve aplicar intervenções de autocontrole de forma indiscriminada. Antes, porém, elas devem respeitar as características dos indivíduos (Rosa, Olivares, \& Sánches, 1999 ).

Um movimento que vem ganhando aceitabilidade na comunidade analítico-comportamental é uma teoria pós-skinneriana para o comportamento verbal chamada Teoria dos Quadros Relacionais (Relational Frame Theory [RFT]). Ela constrói pressupostos sobre a linguagem e a cognição de forma precisa e com solidez empírica (Hayes, Pistorello, \& Biglan, 2008). A teoria trata da "aprendizagem relacional". Responde-se a eventos por meio de outros e, usando-se "quadros relacionais", alguém pode responder a um evento como equivalente ou oposto a outro evento. Assim, funções de estímulos novos são adquiridas nessas relações. Os quadros relacionais mais básicos são: igualdade, comparação, oposição, distinção, espaciais, temporais, de hierarquia, de causalidade, dêiticos e de perspectiva (Boavista, 2015).

Esses processos são responsáveis por habilidades humanas no campo da lógica e resolução de problemas que, embora úteis, podem levantar controvérsias. É indesejável que alguns hábitos tradicionais, muitas vezes até essenciais, sejam suprimidos:

Uma simples linha de coordenação permite aos humanos se lembrarem de situações

[. . .], mas isso pode significar a transposição de eventos dolorosos para qualquer situ- 
ação e em qualquer período de tempo [. . .]. Um quadro de comparação possibilita aos resultados relativos serem balanceados com êxito, mas também possibilitam o indivíduo a se comparar com um ideal irreal [. . .]. Um quadro temporal permite a previsão de resultados, mas também dá margem para que pessoas temam um futuro improvável ou vivam em um passado irresoluto ao ponto de anularem o presente. (Hayes et al., 2008, p. 83).

Logo, do responder relacional podem nascer alguns processos psicológicos que são considerados subjacentes à psicopatologia, e o conjunto deles é chamado de inflexibilidade psicológica. São eles: 1) predominância do passado conceitual e de um futuro temido, baixo conhecimento; 2) falta de clareza de valores e predominância da influenciabilidade e da esquiva de situações; 3) inércia, impulsividade e persistência na esquiva; 4) atrelamento ao eu-conceitual; 5) fusão cognitiva; e 6) esquiva de experiência (Hayes et al., 2008, 2008). Quem age em acordo com esses processos acaba tentando controlar e eliminar emoções, lembranças e quaisquer estímulos negativos, por considerá-los responsáveis pelo seu sofrimento (Abreu, 2006).

Por outro lado, a flexibilidade psicológica é um modelo de funcionamento psicológico que promove saúde e pode ser descrita como:

a habilidade de um ser humano consciente em experienciar por completo os resultados emocionais e cognitivos e em persistir e alterar seu comportamento em prol de valores escolhidos. (Hayes et al., 2008, p. 84).

Quem age ajustado a esses processos se abre para possíveis contatos com fontes novas de reforçamento, pois o controle de estímulos privados que estava sendo problemático é quebrado (Abreu, 2006).

Para alcançar a flexibilidade psicológica, um modelo de intervenção clínico foi desenvolvido: a Terapia da Aceitação e Compromisso (ACT). O fomento dos processos de conscientização e aceitação, bem como os de mudança de comportamento e compromisso são complementares e são o objetivo da intervenção (Hayes et al., 2008; Saban, 2015). A ACT leva a pessoa a atuar com responsabilidade para com suas escolhas e a aceitar os eventos privados como aparecem (Soriano \& Salas, 2006; Saban, 2015).

O uso dos procedimentos que levem aos processos de aceitação e compromisso permite ligar comportamentos a valores. Comportamentos que antes eram controlados pela esquiva dos eventos privados ficam abertos para a ampliação do repertório comprometido com valores pessoais. O esforço para se livrar de eventos privados avaliados como negativos é substituído pelo esforço para alcançar consequências reforçadoras definidas individualmente (Twohig, Shoenberger, \& Hayes, 2007).

Por exemplo, trabalhando em pacientes com diabetes tipo 2, Gregg, Callaghan, Hayes e Glenn-Lawson (2007 apud Hayes et al., 2008) constataram que a ACT, combinada com automonitoramento, foi eficaz no autocontrole de níveis de glicose sanguínea. Além de resultar em mudanças no autocontrole, trouxe aumento na flexibilidade psicológica, sobretudo quanto aos pensamentos e sentimentos negativos associados ao diabetes. Nesse caso, em vez de tentar evitar os sentimentos referentes à doença, aumenta-se o autocontrole em relação aos comportamentos alimentares. 
Assim, a ACT promove o autocontrole por meio de padrões mais amplos de comportamentos orientados para os valores individualmente identificados (values-driven actions). Embora essa intervenção esteja configurada na prática clínica, o modo como ela age é familiar ao OBM, pois inclui desenvolver metas concretas em áreas específicas, sendo que os comportamentos ligados a essas metas estão envolvidos em relações de longo prazo (Bond, Hayes, \& Barnes-Holmes, 2006). Também algumas doenças já consideradas de certa forma até comuns em contextos organizacionais têm sido tratadas com eficácia pelo modelo, como: depressão, estresse, síndrome de Burnout, ansiedade, psicose, dor, tratamento de doenças, controle de peso, estigma e o fumo. Dessa forma, a ACT está ligada às organizações e ao trabalho (Hayes et al., 2006 apud Hayes et al., 2008).

O que se apreende disso é a necessidade de parar de gastar tanto tempo na remoção de eventos aversivos e atentar aos reforçadores a longo prazo, ou aos valores (Hayes et al., 2008).

Perlmuter e Langer (1979 apud Vandenberghe \& Assunção, 2009) fizeram um estudo para verificar se há uma tendência de melhor funcionamento psicológico nas pessoas mais atentas às suas escolhas diárias. Quatro grupos participaram do estudo. No primeiro, os participantes deveriam monitorar uma única atividade própria. No segundo, a atividade automonitorada mudava diariamente, de acordo com as requisições dos pesquisadores. $\mathrm{O}$ terceiro grupo faria a mesma coisa que o segundo, mas especificando como realizaram a tarefa e criando mais três formas alternativas de realizá-la. O quarto grupo deveria fazer a mesma coisa que o terceiro, com a diferença de que eram eles, e não os pesquisadores que escolhiam qual atividade monitorar. Os resultados indicaram que o quarto grupo, o que teve que prestar mais atenção a suas atividades, saiu-se melhor que os outros em medidas de depressão, independência, confiança, atenção concentrada e diversificação das atividades (criatividade).

Uma noção relacionada com a atenção vem sendo recentemente enfatizada nas terapias comportamentais e cognitivas, dentre elas, a ACT. Trata-se do mindfulness, que pode ser entendido como atenção e consciência plena ou presença atenta e reflexiva (Pareja, 2006). Tem origem na tradição de meditação oriental, mas já perdeu sua conotação religiosa, estando incorporada ao escopo de técnicas das terapias comportamentais e cognitivas. A noção se contrapõe ao fenômeno de mindlessness, que descreve a vivência da pessoa como se ela estivesse "em piloto automático", confiando em visões únicas previamente estabelecidas (Vandenberghe \& Assunção, 2009). "As conotações psicológicas do termo são evidentes" (Pareja, 2006, p. 92), uma vez que a técnica permite ao praticante centrar-se no momento presente, abrindo-se a experiências e atos, aceitando-os radicalmente.

A técnica para alcançar o mindfulness consiste na pessoa observar, sem controlar, suas respostas fisiológicas, aceitando mudanças na sensação e no movimento. Tenta-se, assim, sentir e conhecer tudo o que acontece com ela, em um interesse ativo, sem lutar contra pensamentos e sentimentos que julgue desagradáveis. Se eventos desagradáveis ocorrem, a pessoa simplesmente os aceita e volta a fazer o que estava fazendo (Pareja, 2006). Espera-se que os resultados dessas intervenções promovam "um estilo de vida em que a pessoa faz o que valoriza em vez de se trancar em padrões defensivos 'dentro da sua cabeça'" (Pareja, 2006, p. 98). Falar em padrões "dentro da cabeça" é falar sobre eventos privados. 
Parte importante dos eventos estão escondidos debaixo da pele de cada um. Para a Análise do Comportamento, os eventos privados não têm natureza diferente dos públicos, eles existem e fazem parte de relações comportamentais. A diferença entre sentimentos, pensamentos e comportamentos motores como andar, por exemplo, é que os dois primeiros ocorrem debaixo da pele e, por isso, só podem ser acessados pelo próprio organismo, enquanto que o último pode ser acessado por outros (Simonassi, Tourinho, \& Silva, 2001).

A RFT joga uma luz sobre como o comportamento verbal e os eventos privados têm papel na gênese da saúde e da doença nessa configuração, mas vai além quando coloca os valores como parte da mudança. Isso porque o autocontrole está em íntima relação com aquilo que chamamos de ética e moral. Os conflitos de contingências que caracterizam uma situação de autocontrole podem também ser conflitos morais. O comportamento moral e ético, assim como outros quaisquer, é atribuído às contingências ambientais, porém, grande parte dessas contingências está na comunidade verbal da qual a pessoa faz parte: "A comunidade verbal fornece muito cedo no processo de socialização da criança uma infinidade de convenções sobre o que é certo e o que é errado" (Simonassi et al., 2001, p.54). Se, como já vimos, o autocontrole acontece em situações de conflito de contingências, muitos desses conflitos estarão fundamentados nas regras sociais e nas autorregras que aprendemos a formular (Prust \& Gomide, 2007).

\section{Autocontrole na Humanização dos Contextos Organizacionais}

Como humanização nos contextos organizacionais entende-se a realização de uma visão que enxerga o performer como um investimento, sendo alguém capaz de receber um tratamento civilizado em estilos de gestão e supervisão inerentemente respeitosos para com o indivíduo. Essa visão contrasta com aquela que encara o performer com uma engrenagem na máquina organizacional, cumprindo uma função necessária, em ambientes carregados de punição e adversidades (Crowell \& Anderson, 2005).

A relação do autocontrole com a humanização do local de trabalho e das organizações em geral vem associada a uma argumentação mais complexa. Por isso, listamos três pontos pelos quais ela passa. O primeiro refere-se ao papel da Análise do Comportamento na Psicologia em geral. Esse papel, por sua vez, leva ao segundo ponto, que é a demanda pelo reconhecimento do reforço positivo (Geller, 2005 apud Crowell \& Anderson, 2005). A partir dessa proposta, surge o terceiro ponto, quando Crowell e Anderson (2005) listam 10 motivos pelos quais o OBM deve ser reconhecido como uma abordagem que humaniza o ambiente de trabalho, sendo que o último desses motivos é que a disciplina promove o autocontrole, e que isso vem como consequência, muitas vezes indireta, de suas práticas e técnicas.

Quanto ao primeiro ponto, a American Psychology Association (APA) fez uma campanha de educação pública para que a década de 2000 fosse conhecida como a Década do Comportamento, assim como a década de 1990 foi conhecida como a Década do Cérebro. A iniciativa multidisciplinar, com amplo apoio da comunidade científica, se concentrou em responder a desafios da sociedade nas áreas de saúde, segurança, educação, prosperidade e democracia, pelas ciências comportamentais e sociais. Dentro desse movimento, a Análise Aplicada do Comportamento e o OBM, que poderiam ter algum papel, foram negligenciados, e ambos continuam ainda hoje gozando de uma obscuridade, quando comparados a 
Psicologia nas Organizações tradicional e a Psicologia Positiva (Geller, 2003 apud Crowell \& Anderson, 2005).

A Análise do Comportamento é ativa e produtiva em termos de pesquisa, o que pode ser observado nas reuniões da Association for Behavior Analysis (ABA), bem como noutros encontros, regionais, nacionais e internacionais (Neuringer, 1984). Dentre os dados que indicam um crescimento da área está o número de membros da ABA. Em sua fundação, contava com 900 membros, após 35 anos, 4500 membros. Embora esse número pareça pouco se comparado aos 160000 membros da APA, vale lembrar que a Análise do Comportamento nunca fez parte do mainstream da Psicologia norte-americana, ainda que B. F. Skinner seja considerado um dos psicólogos mais influentes do século XX (Cunha \& Verneque, 2004; Malott, 2005).

Na realidade, alguns princípios básicos da Psicologia Operante foram aceitos por grande parte da Psicologia, independentemente da abordagem como, por exemplo, a noção de reforçamento, que também está presente na Psicologia Cognitiva, porém com críticas e outras interpretações. Parte da crítica gira em torno dos limites e da abrangência desses princípios em contextos abertos ou sociais comuns (Frutuoso \& Cirino, 2006). Ou seja, o que se discute não são seus princípios, mas sim o quanto do comportamento humano está sob controle deles e a interpretação que Ihes é dada. Para a comunidade analítico-comportamental, todavia, são esses princípios que devem orientar e permitir o melhor tratamento de quaisquer questões humanas a serem resolvidas.

No entanto, basear a relevância de uma pesquisa básica no seu uso atual na sociedade pode não ser o melhor caminho. Por outro lado, não podemos fazer de conta que não há contingências sociais envolvidas na cultura científica. O comportamento do cientista não escapa das relações de dependência com outros eventos, do contrário, ele produz reforçadores poderosos, tanto dentro do laboratório, quanto na cultura da qual faz parte. Dentro do laboratório, os repertórios de comportamento científicos persistentes são mantidos por consequências intrínsecas ao trabalho científico analítico-comportamental, uma vez que a investigação operante pode ser descrita como relativamente fácil de ser realizada, há regularidades nos dados, é nomológica [lawfullness], além de ser divertido para alguns (Neuringer, 1984). Sidman (2008) explicita essa relação quando diz acreditar que a pesquisa básica tem a função de produzir conhecimento aplicável. Assim, a pesquisa é apoiada porque o conhecimento é socialmente valorizado em si pelo seu potencial de produção:

As consequências da pesquisa básica, que chamamos de "conhecimento", se tornaram valiosas porque têm levado aos muitos reforçadores subsequentes que a aplicação tem tornado possíveis. Conhecimento se tornou, por meio disso, um reforçador generalizado em nossa comunidade. Pesquisadores comportamentais em pesquisa básica que desvalorizam a pesquisa aplicada e a aplicação ignoram princípios básicos que explicam tanto porque a sociedade continua apoiando pesquisas desenvolvidas inicialmente só para produzir mais conhecimento quanto porque a sociedade não mantém seu apoio à ciência cuja produção julga estéril. (Sidman, 2008, p. 9).

Historicamente, pesquisa básica em Análise do Comportamento está associada com a pesquisa aplicada. Pesquisadores básicos como Skinner, Keller, Fester e Azrin também trabaIhavam na aplicação da ciência (Neuringer, 1984). Hoje, a referência à pesquisa experimental 
continua sendo imperativa, não apenas pelo manto de respeito que ela pode fornecer - e que os profissionais da área anseiam tanto - mas também porque esse é o seu principal argumento. O pretexto que os consultores em OBM têm para desenvolver seus trabalhos é que ele se fundamenta em pesquisas científicas de base e que, nesse sentido, está à frente da concorrência. Por isso, pode se prejudicar se sua prática não fizer referência à pesquisa laboratorial e à teoria analítico-comportamental (Malott, 2005).

Ainda assim, não há o reconhecimento de conceitos analítico-comportamentais como o reforço positivo, mesmo que esse tenha mostrado bastante eficácia em melhorar a qualidade de vida, seja no trabalho ou em outros contextos. O OBM poderia reforçar sua caixa de ferramentas, como também promover o seu próprio reconhecimento e aceitação na Psicologia mainstream (Geller, 2003 apud Crowell \& Anderson, 2005). Para alcançar esse objetivo, o profissional deve usar reforço positivo como pedra angular para a prática profissional (Wiegand \& Geller, 2005 apud Crowell \& Anderson, 2005). A utilização do reforço positivo como preferencial a formas de coerção tem sido amplamente comentada na Análise do Comportamento (Sidman, 2003). Yáber-Oltra (2004) ilustra bem esse ponto quando, sobre os méritos do OBM, afirma:

O interesse consiste em fixar condições para que o trabalho se realize sob mínimas condições aversivas, nas máximas condições de reforçamento positivo e eliciando emoções positivas para que o desempenho laboral ocorra e se mantenha com intensidade (p. 50).

Finalmente, chegamos ao terceiro ponto. Crowell e Anderson (2005), ampliando essa discussão, listam outras 10 maneiras que distinguem o OBM como uma abordagem "positiva", com capacidade de humanizar as práticas de gestão: 1) orientação para o desenvolvimento; 2) foco no processo de desenvolvimento do desempenho; 3) reconhecimento das diferenças individuais; 4) responsabilidades baseadas no controle pessoal; 5) ênfase na definição e clarificação do trabalho; 6) aumento da conscientização do próprio comportamento e de seus resultados; 7) utilização de metas para moldar e refinar o sucesso pessoal; 8) gestão por reconhecimento ao invés de gestão por exceção; 9) incentivo a melhores taxas de desempenho em lugar de incentivo a melhores pessoas; e 10) promoção do autogerenciamento.

A promoção do autogerenciamento, ou como estamos chamando ao longo desse trabaIho, do autocontrole, se fundamenta no que talvez seja o resumo do que vem sendo nomeado como tratamento humano e com civilidade no local de trabalho: oferecer circunstâncias e ferramentas para que os performers possam controlar, eles mesmos, o seu desenvolvimento e o seu destino (Geller, 2002, apud Crowell \& Anderson, 2005).

\section{Considerações Finais}

Esse trabalho realizou uma exploração e descrição da literatura analítico-comportamental do autocontrole relacionada a um contexto específico: o setting organizacional. O uso de técnicas de autocontrole nas organizações foi recomendado, identificando suas contribuições e situando sua utilização na promoção da saúde do trabalhador e na humanização da organização de trabalho. O autocontrole como a aplicação deliberada de princípios de mudança comportamental a si mesmo, e o OBM como a área de estudo e aplicação da filosofia do Behaviorismo Radical às organizações. No ponto de intersecção dessas noções, estão desde modelos construídos especificamente com esse objetivo até a posição ocupada pelo con- 
ceito na humanização dos contextos organizacionais, passando pela saúde do trabalhador, e o quanto técnicas tradicionais das aproximações analítico-comportamentais à área contribuem na promoção de pessoas com maior capacidade de controlar suas próprias vidas.

Considera-se que uma preocupação maior com o conceito pela Análise do Comportamento pode trazer à comunidade de cientistas, pesquisadores e profissionais pautados na filosofia do Behaviorismo Radical maior aceitabilidade de seu projeto científico, tanto na Psicologia em geral, como na esfera social e da saúde. Também se sugere um maior esforço por parte dos pesquisadores da área em fundamentar empiricamente a posição de que as técnicas tradicionais do OBM colaboram com a promoção de indivíduos mais autônomos e com a promoção de saúde, além de acrescer um testemunho às práticas de gestão, sobretudo no nível da gestão estratégica.

Por fim, e a partir das discussões realizadas por Zanelli (2016) quanto aos temas transdisciplinares em Saúde, salientamos os fatores multideterminantes da constituição da saúde no trabalho, destacando as relações e a organização como elementos fundamentais à sua promoção ou perda. Mais um ponto que merece atenção tanto na pesquisa quanto na intervenção, pois se relaciona à formação das práticas de prevenção. Nessa direção, cabe dotar as pessoas de recursos para lidar com variáveis intrapessoais e enfrentar condições externas tende a possibilitar êxito nas ações.

\section{Referências}

Abreu, P. R. (2006) Terapia analítico-comportamental da depressão: Uma antiga ou uma nova ciência aplicada? Revista Psiquiatria Clínica, 33(6), 322-328.

Boavista, R. R. C. (2015). Teoria das molduras relacionais. In Lucena-Santos, P., Pinto-Gouveia, J., Oliveira, M. S. (Orgs.). Terapias comportamentais de terceira geração: Um guia para profissionais (pp. 151-178). Novo Hamburgo, RS: Sinopys.

Bond, F. W., Hayes, S. C., \& Barnes-Holmes, D. (2006). Psychological flexibility, ACT and organizational behaviour. Journal of Organizational Behavior Management, 26, 25-54.

Casseb, M. S., Bispo, M. S. M., \& Ferreira, E. A. P. (2008). Automonitoração e seguimento de regras nutricionais em diabetes: dois estudos de caso. Interação em Psicologia, 12(2), 223-233.

Crowell, C. R., \& Anderson, D. C. (2005). On reinventing OBM: Comments regarding Geller's proposals for change. Journal of Organizational Behavior Management, 24(1-2), 27-53.

Cunha, R. N., \& Verneque, L. P. S. (2004). Notícia: Centenário de B. F. Skinner (1904-1990): Uma ciência do comportamento humano para o futuro do mundo e da humanidade. Psicologia: Teoria e Pesquisa, 20(1), 93-94. Disponível em <http://www.scielo.br/scielo. php?script=sci_arttext\&pid=S0102-37722004000100014\&Ing=en\&nrm=iso >.

Frutoso, J. T.; \& Cirino, S. D. (2006). A aplicação dos princípios do comportamento em contextos abertos. Revista de Ciências Humanas, 40, 339-360. Disponível em <https://periodicos.ufsc.br/index.php/revistacfh/article/view/17656/16216>.

Hayes, S. C., Pistorello, J., \& Biglan, A. (2008). Terapia de aceitação e compromisso: Modelo, dados e extensão para a prevenção do suicídio. Revista Brasileira de Terapia Comportamental e Cognitiva, 10(1), 81-104. Disponível em <http://pepsic.bvsalud.org/ scielo.php?script=sci_arttext\&pid=S1517-55452008000100008>. 
Jacques, M. D. G. C. (2003). Abordagens teórico-metodológicas em saúde/doença mental \& trabalho. Psicologia \& Sociedade, 15(1), 97-116.

Kerbauy, R. (2009). História de vida. Boletim Paradigma, 04, 28-30.

Malott, R. Entrevista realizada no Hotel Hilton, Chicago, EUA, em 28/05/2005. Disponível em $<$ http://dick-malott.com/behaviorism/spanishinterview />.

Neuringer A. (1984). Melioration and self-experimentation. Journal of Experimental Behavior Analysis, 42(3), 397-406.

Pareja, M. A. V. (2006). Mindfulness. Papeles del Psicólogo, 27(2), 92-99.

Prust, L. W., Gomide, P. I. C. (2007). Relação entre comportamento moral dos pais e dos filhos adolescentes. Estudos de Psicologia, 24(01), 53-60.

Rosa, A., Olivares, J., \& Sánches, J. (1999). La terapia de conducta en el contexto espanõl e internacional: Situación actual y fatores implicados en su eficacia. Psicologia Conductual, 7(2), 215-252.

Saban, M. T. (2015). O que é terapia de aceitação e compromisso? In: Lucena-Santos, P., Pinto-Gouveia, J., \& Oliveira, M. S. (Orgs.). Terapias comportamentais de terceira geração: Um guia para profissionais (pp. 179-216). Novo Hamburgo, RS: Sinopys.

Sidman, M. (2003). Coerção e suas implicações. Campinas, SP: Editorial Psy.

Sidman, M. (2008). Symmetry and equivalence relations in behavior. Cognitive Studies, 15, 322-332.

Simonassi, L. E., Tourinho, E. Z., \& Silva, A. V. (2001). Comportamento privado: Acessibilidade e relação com comportamento público. Psicologia: Reflexão e Crítica, 14(1), $133-$ 142. Disponível em <http://www.scielo.br/scielo.php?script=sci_arttext\&pid=S010279722001000100011\& Ing=en\&nrm=iso>.

Skinner, B. F. (1953). Ciência e comportamento humano. New York: The Free Press.

Soriano, M. C. L., Salas, M. S. V. (2006). La terapia de aceptación y compromiso (act): Fundamentos, características y evidencia. Papeles del Psicologo, 272, 79-91.

Twohig, M. P., Shoenberger, D., \& Hayes, S. C. A. (2007). Preliminary investigation of acceptance and commitment therapy as a treatment for marijuana dependence in adults. Journal of Applied Behavior Analysis, 40(4), 619-632.

Vandenberghe, L., \& Assunção, A. B. (2009). Concepções de mindfulness em langer e KabatZinn: Um encontro da ciência ocidental com a espiritualidade oriental. Contextos Clínicos, 2(2), 124-135.

Yáber-Oltra, G. (2004). Cambio organizacional integral com gerencia de sistemas conductuales. Analogias del Comportamiento: Boletín de La Escuela de Psicologia de la UCAB, 7, 47-74.

Zanelli, J. C. (2016). Prefácio: Sobre os temas transdisciplinares em Saúde Mental e Trabalho. In Macêdo, K. B. et al. (Orgs.) Organização do trabalho e adoecimento - Uma visão interdisciplinar. Goiânia: Ed. da PUC Goiás.

Recebido: 01/05/2017

Última revisão: 27/07/2017

Aceite final: 07/08/2017 


\section{Sobre os autores:}

Eveli Freire Vasconcelos: Graduação, mestrado e doutorado em Psicologia pela Universidade Católica Dom Bosco (UCDB). Atua como docente e supervisora de estágio Curso de Graduação em Psicologia. É coordenadora do curso de Pós-Graduação Lato Sensu em Psicologia Organizacional e do Trabalho (EAD e Presencial) da UCDB. Tem experiência em Psicologia, Organizações e Trabalho, atuando principalmente em temas da área: gestão de pessoas, comportamento organizacional. E-mail: evelivasconcelos@yahoo.com.br

Gerson Armênio Fabrício Bauermeister: Graduado em Psicologia pela Universidade Católica Dom Bosco (UCDB). Psicólogo da Assembleia Legislativa do Estado de Mato Grosso. E-mail: senhordopantanal@hotmail.com

Heloisa Bruna Grubits Freire: Doutora em Ciências Biomédicas, pela Universidade Estadual de Campinas (UNICAMP). Professora titular da Universidade Católica Dom Bosco (UCDB) e Coordenadora do Programa de Equoterapia (PROEQUO-UCDB). É líder do grupo de pesquisa CNPQ Equoterapia: teoria e técnica. Atua nos seguintes campos: Psicologia da Saúde, Saúde Mental de Estudantes, Equoterapia e Equitação Terapêutica. Tem pesquisado os seguintes temas: Equoterapia e Equitação Terapêutica; Relação interespécie, Terapia mediada por cães e gatos, inserção de cães nas práticas pedagógicas dos cursos de graduação em Psicologia, Saúde Mental, Qualidade de Vida e Coping em Estudantes Universitários. E-mail: grubitshb@hotmail.com

Thaize de Souza Reis: Graduação em Psicologia, Mestrado em Educação Especial e Doutorado em Psicologia pela Universidade Federal de São Carlos (UFSCar). Professora Adjunta da Universidade Federal de Mato Grosso do Sul. E-mail: thaizereis@gmail.com 
